\title{
Una Propuesta de Sistema de Diagnóstico de Fallos Robusto Ante la Presencia de Pérdida de Información y Ruido en Sistemas Mecánicos
}

\author{
Ortiz Ortiz, Francisco Javier $^{1}$ iD ; Llanes-Santiago, Orestes $^{2, *}$ \\ ${ }^{1}$ Universidad Politécnica Salesiana,Programa de Maestría en Electrónica y Automatización, Cuenca, Ecuador \\ ${ }^{2}$ Universidad Tecnológica de La Habana José Antonio Echeverría,CUJAE, Dpto. de Automática y Computación, La Habana, Cuba
}

\begin{abstract}
Resumen: Hoy en día, es un requisito de la industria moderna lograr elevados rendimientos económicos con un aumento continuo de la calidad de los productos finales, tener elevados niveles de seguridad industrial y reducir al mínimo las posibles afectaciones al medio ambiente; todo lo cual hace necesario la rápida detección e identificación de los fallos que se presenten en los sistemas industriales. La evolución de la Internet de las cosas y los avances tecnológicos en los medios técnicos de automatización, las redes industriales y las comunicaciones inalámbricas entre otros elementos, han permitido un crecimiento significativo del número de herramientas a usar para el tratamiento y gestión de la información obtenida por los sistemas de supervisión, control y adquisición de datos (SCADA) de los procesos industriales. Sin embargo, el desempeño de estas herramientas y en especial de los sistemas de diagnóstico de fallos se ve afectado por dos problemas concretos: la presencia de ruido en las mediciones y la pérdida de información de variables medidas. En el presente trabajo se propone una metodología para el diagnóstico de fallos en sistemas industriales mecánicos utilizando herramientas de inteligencia computacional que logra un comportamiento robusto ante la presencia de pérdida de información y el ruido logrando altos niveles de desempeño. La metodología propuesta se aplica al problema de prueba DAMADICS que representa a una válvula electro-neumática que es un tipo de actuador muy utilizado en los sistemas industriales modernos. Los resultados satisfactorios que se obtienen demuestran la efectividad y validez de la propuesta.
\end{abstract}

Palabras claves: Diagnóstico de fallos, pérdida de datos, ruido, imputación de datos, procesos industriales, inteligencia computacional

\section{A Proposal of Robust Fault Diagnosis System in Presence of Missing Data and Noise in Mechanical Systems}

\begin{abstract}
Currently, the modern industry requires to achieve high economic returns with a continuous increase in the quality of the final products, to have high levels of industrial safety and to minimize possible effects on the environment. For accomplishing these requirements, it is necessary a fast detection and identification of faults that occur in the industrial systems. The evolution of the Internet of things and technological advances in automation devices, industrial networks and wireless communications, among other elements, have allowed a significant growth in the number of tools to be used for the treatment and management of the information obtained from the industrial processes by the supervision, control and data acquisition systems (SCADA). However, the performance of these tools, and especially of the fault diagnosis systems, are affected by two specific problems: the presence of noise and missing information on the measured variables. In this paper, a novel methodology for fault diagnosis in mechanical industrial systems is proposed by using computational intelligence tools. The proposal presents a robust behavior in presence of missing data and noise in the measurements by achieving high levels of performance. The proposed methodology is applied to the DAMADICS actuator FDI benchmark which is an electro-pneumatic valve widely used in modern industrial systems. The satisfactory results obtained demonstrate the effectiveness and validity of the proposal.
\end{abstract}

Keywords: Fault diagnosis, missing data, noise, data imputation, industrial processes, computational intelligence

\section{INTRODUCCIÓN}

En la actualidad los sistemas industriales se encuentran en constante proceso evolutivo, y ya es cotidiano el uso de términos como industria inteligente y cuarta revolución industrial o industria 4.0 (Ustundag \& Cevikcan, 2017). Estos términos hacen referencia a la interconexión de todos los componentes de una industria para conseguir un funcionamiento automatizado efectivo que logre 
elevados rendimientos económicos con un aumento continuo de la calidad de los productos finales, tener elevados niveles de seguridad industrial y reducir al mínimo las posibles afectaciones al medio ambiente (Zabiński et al., 2019). Para lograr la anterior se necesita la detección y localización temprana de los fallos que comúnmente aparecen en los sistemas industriales en sensores, actuadores y/o en los procesos ya que los mismos se traducen directamente en problemas de seguridad para los operadores, posibles afectaciones al medio ambiente y pérdidas económicas. Estas razones han motivado el desarrollo de un gran número de investigaciones en el campo del diagnóstico de fallos en procesos industriales en las últimas décadas. (Chen \& Li, 2018; Rodríguez et. al., 2019; Ahmed et al., 2021).

Los sistemas mecánicos son parte fundamental de cualquier industria y sobre todo de la industria moderna de manufactura y es precisamente en estos sistemas mecánicos, donde se presenta una gran parte de los fallos que ocurren en los sistemas industriales (Rodríguez et. al., 2019; Aydin et al., 2014).

En general, las metodologías propuestas en la literatura científica para el diagnóstico de fallos pueden agruparse en dos grandes grupos: los métodos basados en modelos (Camps-Echevarría et al., 2010; Rivera et al., 2018) y los métodos basados en datos históricos (Rodríguez Ramos et. al., 2019; Llanes-Santiago et. al., 2018). En el primer grupo, es necesario el uso de modelos que representen los diferentes modos de operación de los procesos. Las herramientas establecidas en este enfoque se basan en la generación de residuos que se generan de la diferencia entre las mediciones que se obtienen directamente del proceso real y los valores obtenidos del modelo de dicho proceso. La gran dificultad en la aplicación de las metodologías basadas en modelos es que se necesita un elevado conocimiento de las características del proceso, sus parámetros y zonas de operación y en la actualidad eso es difícil lograrlo por la elevada complejidad de las plantas industriales modernas. Por otra parte, los métodos basados en datos no necesitan de un modelo matemático preciso y no requieren de un elevado conocimiento inicial de los parámetros del proceso (Prieto-Moreno et al., 2015; Cerrada et al., 2018). En ese sentido, existen múltiples propuestas que utilizan herramientas computacionales y matemáticas como: lógica difusa (Rodríguez Ramos et. al., 2019), agrupamiento (Cerrada et al., 2016; Rodríguez et. al., 2019), herramientas estadísticas (Rezamand et. al., 2020; Prieto-Moreno et al., 2015) y redes neuronales (Saufi et. al., 2020) por solo mencionar algunas.

Entre los principales factores que afectan seriamente a las metodologías de diagnóstico de fallos basados en datos históricos se encuentran los datos fuera de rango, los ruidos en las mediciones y la pérdida de información todo lo cual es muy común en los ambientes industriales. De lo anterior surge la necesidad de atender esta problemática para que el diagnóstico de fallos sea robusto, evitándose falsas alarmas y la pérdida de confiabilidad del sistema (Askarian et al., 2016).

Las observaciones con valores incompletos pueden deberse a diversas fuentes como fallos en los sensores, problemas de funcionamiento ocasionales de los sistemas de adquisición de datos, posi- bles errores en las redes de trasmisión de datos y en los protocolos de comunicación por solo mencionar los más comunes. Varios métodos para el tratamiento de los datos perdidos se han propuesto en la literatura científica (Askarian et al., 2016; Laencina et al., 2010; Sovilj et al., 2016). Los principales son (Laencina et al., 2010):

- Ignorar y eliminar los datos incompletos: se usan solo los datos que estén completos.

- Imputar o estimar los datos perdidos mediante herramientas estadísticas o de inteligencia computacional.

- Modelo basado en la distribución de los datos.

- Máquinas de Aprendizaje: procedimientos donde los valores perdidos se incorporan al clasificador.

Cuando se realiza una revisión de las técnicas más utilizadas para tratar los datos perdidos se llega a la conclusión que son los dos primeros métodos mencionados anteriormente los más usados. Sin embargo la información perdida puede ser importante para descubrir posibles relaciones entre las variables y condiciones anormales de operación (Askarian et al., 2016). Es por esta razón que lo más recomendado para el tratamiento de los datos perdidos en el caso del diagnóstico de fallos es el de imputar. Con este proceso se estiman los valores perdidos usando toda la información disponible y no se eliminan observaciones que pueden contener información importante para la labor de diagnóstico (Askarian et al., 2016; Llanes-Santiago et. al., 2018).

Son múltiples los trabajos encontrados que abordan las técnicas basadas en imputación o estimación de parámetros (Askarian et al., 2016; Laencina et al., 2010; Sovilj et al., 2016). Sin embargo, todos los métodos realizan la imputación utilizando el conjunto de datos completo o parte del mismo y no se establecen requerimientos estrictos de tiempo.

En el caso de los sistemas industriales, las exigencias son muy elevadas. La imputación de las variables perdidas de una observación obtenida hay que hacerla en línea y con requerimientos de tiempo estrictos determinados por el período de muestreo que se ha establecido en el sistema de adquisición de datos. Además, hay que tener en cuenta que todas estas mediciones están afectadas por el ruido que es característico en los procesos industriales.

En los últimos años las redes neuronales de aprendizaje profundo han ido incrementando su popularidad por su capacidad de aprender características no lineales complejas que permiten mejorar significativamente el poder discriminativo en los procesos de clasificación. En Eren \& Kiranyaz (2019), y Lee et. al. (2019) se propone el uso de redes neuronales convolucionales (CNN) que aprenden características altamente discriminantes directamente de los datos del sensor de entrada sin procesar, logrando un rendimiento competitivo con una configuración CNN simple y compacta. En Medina et. al. (2019), Chen et al. (2021) y Xiao et. al. (2019) se propone el uso de redes neuronales de aprendizaje profundo por su facilidad para aprender características importantes de señales sin un procesamiento previo. En Rezamand et. al. (2020) se propone el uso de la transformada wavelet para la detección de fallos en señales de vibración con un sistema 
híbrido de detección de fallos basado en una combinación de una red neuronal de regresión generalizada para imputación única (GRNN-ESI), análisis de componentes principales (PCA) y función de densidad de probabilidad basada en wavelets (PDF). Existen otras propuestas de combinación de diferentes herramientas para el análisis de señales para mejorar el rendimiento de los sistemas de detección de fallos, como el caso de Li et. al. (2021) donde se propone un método para crear modelos de detección de fallos solo a partir de datos normales para la detección de fallos en impresoras 3D.

El objetivo principal de este trabajo es proponer una metodología de diagnóstico de fallos para sistemas mecánicos presentes en la industria basada en un esquema híbrido que combina algoritmos de imputación de fácil implementación con herramientas de aprendizaje profundo. La metodología que se propone realiza la imputación en línea de los datos perdidos logrando elevados niveles de desempeño en la clasificación de los fallos a partir de utilizar una red neuronal de aprendizaje profundo de tipo LSTM (por sus siglas en inglés Long Short Term Memory). La propuesta presenta un comportamiento muy robusto ante la presencia de ruido y las posibles desviaciones que puede introducir el proceso de imputación de los datos perdidos. Lo anterior también constituye la principal contribución de este trabajo.

La organización del artículo es la siguiente: en la sección 2 se presentan: la metodología propuesta, las características generales de las herramientas utilizadas en la misma, el caso de estudio y el diseño de los experimentos. En la sección 3 se realiza el análisis y discusión de los resultados obtenidos. Además, se realiza una comparación de los resultados obtenidos por la propuesta con los obtenidos por otros esquemas de diagnósticos presentados recientemente en la literatura científica. Finalmente, se presentan las conclusiones.

\section{MATERIALES Y MÉTODOS}

\subsection{Mecanismos de pérdida de información}

En Little \& Rubin (2014) se exponen los tres tipos de mecanismos de pérdida de información que se pueden presentar. Los mismos son:

- Pérdida de información completamente aleatoria (MCAR): La probabilidad de que se pierda información de una variable $X$ en una observación no está relacionada con las otras variables medidas ni con los valores de la propia variable $X$. Lo anterior indica que la pérdida de información no depende de los valores de entrada.

- Pérdida de información aleatoria (MAR): La pérdida de información es independiente de las variables perdidas pero el patrón de pérdida de datos se puede predecir a partir del conjunto de las variables.

- Pérdida de información no aleatoria (NMAR): El patrón de datos perdidos no es aleatorio y depende de las variables perdidas.
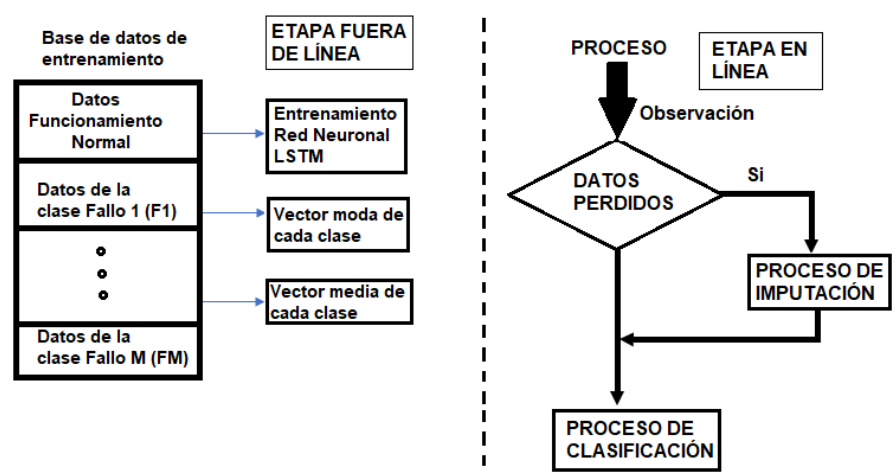

Figura 1. Metodología propuesta para el diagnóstico de fallos con mediciones ruidosas y datos perdidos

En los sistemas industriales el mecanismo de pérdida de información que mayoritariamente se presenta es el MCAR y es el que utilizaremos en este trabajo.

\subsection{Metodología propuesta para el sistema de diagnóstico}

Es conocido de la literatura científica que para la utilización de herramientas de diagnóstico de tipo supervisadas se requiere disponer de una base de datos que contenga la cantidad de observaciones necesarias del estado de funcionamiento normal y de cada uno de los estados de fallo de manera tal que se pueda realizar el entrenamiento de la herramienta a utilizar para lograr los mejores resultados en el proceso de clasificación (Watanabe et al., 1989). Por lo anterior se dispondrá de un matriz de entrenamiento $E \in \mathfrak{R}^{n \times p}$ compuesta de $n$ observaciones de $p$ variables cada una. A cada clase $C_{l} \in \mathfrak{R}^{k \times p}$ para $l=1,2, \ldots, n c$ corresponden $k$ observaciones por lo que $n=n c * k$ donde $n c$ representa el número de clases del sistema.

En la Figura 1 se presenta la metodología propuesta para el diagnóstico de fallos en este trabajo. La misma está compuesta de dos etapas: fuera de línea y en línea.

En la etapa fuera de línea, se realiza el entrenamiento de la red neuronal LSTM. Además, se obtienen los vectores con las medias aritméticas $\left(\bar{X}_{C_{l}}\right)$ y los vectores con los valores de la moda $\left(X_{M C_{l}}\right)$ de las variables de cada clase presente en el sistema las cuales representan el modo de funcionamiento normal y los modos de operación característico de cada uno de los fallos.

En la etapa en línea, cada vez que se recibe una observación se analiza si tiene datos perdidos o no. En caso de no tener datos perdidos se realiza el proceso de clasificación de la observación. En caso de que se detecten variables perdidas se realizará la imputación.

\subsubsection{Proceso de imputación}

Al recibirse una nueva observación con variables perdidas se realiza el siguiente procedimiento para imputar en línea:

1. Se recibe una observación en línea $X \in \mathfrak{R}^{1 \times p}$ con $r$ datos perdidos. Se eliminan las $r$ variables perdidas en la nueva observación y en los vectores de valores medios obtenidos en la 
etapa fuera de línea. Se calcula la distancia de la observación $X \in \mathfrak{R}^{1 \times p-r}$ a los vectores $\bar{X}_{C_{l}} \in \mathfrak{R}^{1 \times p-r}\left(d_{l}=X-\bar{X}_{C_{l}}\right)$ de las clases representadas en la matriz de datos históricos utilizada para el entrenamiento. Se determina la distancia mínima $\left(\min \left(d_{l}\right)\right)$ y con esta, la clase a la que se asignará la nueva observación. La medida de distancia utilizada en este trabajo es la distancia euclideana.

2. Se realiza el proceso de imputación con el método que se haya decidido utilizar.

3. Una vez estimados todos los valores perdidos se clasifica la observación usando la herramienta de clasificación del sistema de diagnóstico.

Observación: Es posible que la herramienta de diagnóstico clasifique a la observación con los datos estimados en una clase diferente a la que fue asignada para realizar el proceso de imputación. La razón de esto se explica en el grado de solapamiento que pueden tener algunas clases en el espacio de las observaciones.

\subsection{Herramientas para la imputación de los datos perdidos}

En este trabajo, se utilizarán dos métodos de imputación: la media aritmética y la moda. Estos métodos fueron escogidos por su demostrada efectividad y la sencillez de su implementación.

Para la imputación de datos perdidos usando la media aritmética se utilizan los valores medios de las variables en cada clase calculados en el proceso fuera de línea $\left(\bar{X}_{C_{l}}\right)$. Es conocido que la media de un conjunto de datos se ve afectada por los datos conocidos como datos fuera de rango. Para evitar esa afectación la base con los datos de entrenamiento debe ser tratada previamente a su uso con alguno de los métodos muy conocidos en ramas matemáticas como la Estadística para la eliminación de este tipo de datos.

Una manera de evitar los efectos que producen los datos fuera de rango es usando el valor de la moda de un conjunto de datos la cual representa el valor de información que más se repite en el conjunto. Usar el valor de la moda permite no tener que hacer un tratamiento de los datos de entrenamiento para eliminar los datos fuera de rango.

Al determinarse en la observación las variables perdidas y una vez clasificada la observación en una clase, se accede al vector de valores medios $\left(\bar{X}_{C_{l}}\right)$ o al vector de valores de la moda de esa clase $\left(X_{M C_{l}}\right)$, según sea el método que se utilice, y se le agrega a la observación el valor correspondiente al valor medio o la moda de cada variable perdida en la posición que le corresponde en la observación.

\subsection{Red Neuronal LSTM}

La red neuronal LSTM pertenece a la familia de las redes neuronales recurrentes (RNN). Son ideales en el aprendizaje, procesamiento y clasificación de datos secuenciales. Ella fue propuesta por Hochreiter y Schmidhuber en Hochreiter \& Schmidhuber (1997) y posteriormente Gers (Ger et. al., 2000)

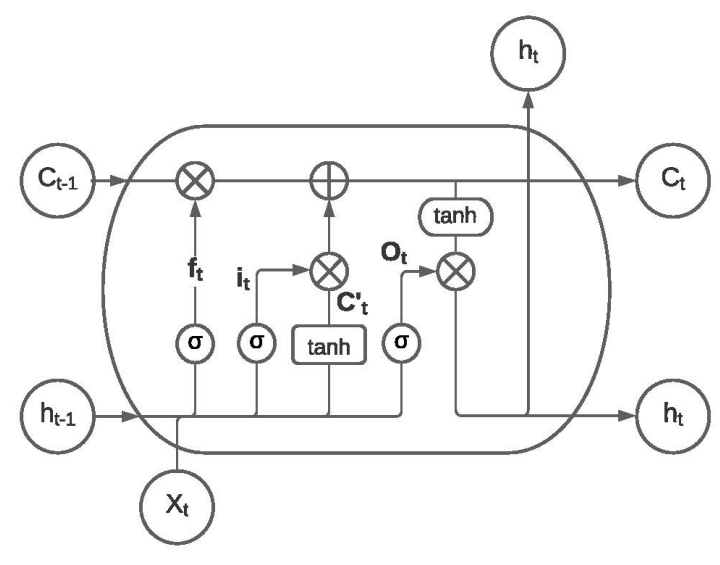

Figura 2. Celda de una red LSTM

propuso una variación que mejoró su funcionamiento. Las redes LSTM tienen como objetivo superar el tema de la desaparición exponencial de los gradientes de error presentes en el entrenamiento por backpropagation de las RNN, utilizando compuertas para retener selectivamente la información que es relevante y olvidando la información que no es relevante. La sensibilidad más baja a la brecha de tiempo hace que las redes $L S T M$ sean mejores para el análisis de datos secuenciales que las RNN.

En las unidades LSTM, cuando los valores de error son propagados de nuevo desde la capa de salida, el error permanece en la celda de la unidad LSTM. Este retorno del error alimenta continuamente el error a cada una de las puertas de la unidad LSTM, hasta que aprenden a eliminar el valor que no es relevante.

Una de las características principales de este tipo de arquitectura de aprendizaje profundo es que puede recordar estados previos, por lo que es un tipo de red muy utilizada en datos de series cronológicas. Las redes LSTM son capaces de aprender dependencias a largo plazo. El modelo almacena la información de la serie de tiempo en la memoria, basada en la importancia expresada por pesos que son aprendidos por el algoritmo. Una diferencia con respecto a las redes neuronales recurrentes (RNN), es el estado de la celda donde se puede agregar o eliminar información a través de estructuras reguladas conocidas como puertas. La arquitectura de la celda $L S T M$ básica se muestra en la Figura 2. Normalmente, un bloque de LSTM tiene una celda de memoria, puerta de entrada, puerta de salida y una puerta de olvido.

La primera etapa conocida como puerta de olvido, decide qué información se desechará. La función $f_{t}$ viene dada por la Ecuación (1), donde $\sigma$ denota la función sigmoidea. La puerta lee $h_{t-1}$ y $x_{t}$ y genera un valor de salida entre 0 y 1 ; donde 0 significa "completamente descartado". y 1 "completamente reservado".

$$
f_{t}=\sigma\left(W_{f} \cdot\left[h_{t-1}, x_{t}\right]+b_{f}\right)
$$

El siguiente paso consiste en decidir qué nueva información se almacenará en la celda de estado indicado como $C_{t}$. Esta tarea es realizada usando dos funciones. La puerta de entrada que es una función sigmoidea que decide qué valores se actualizarán y una función adicional tanh que crea un nuevo vector de valores candi- 


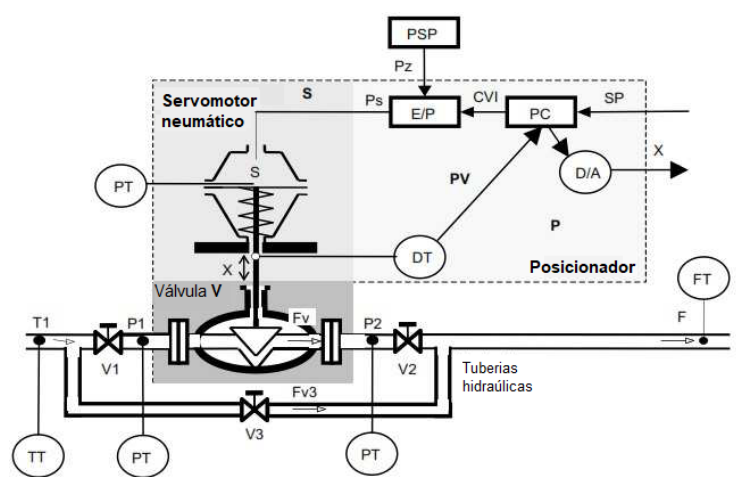

Figura 3. Estructura del actuador Damadics (Bartys et al. (2006))

datos, de acuerdo con las Ecuaciones (2) y (3). En estas ecuaciones, $W$ representa los pesos y $b$ la desviación o bias:

$$
\begin{gathered}
i_{t}=\sigma\left(W_{i} \cdot\left[h_{t-1}, x_{t}\right]+b_{i}\right) \\
C_{t}^{\prime}=\tanh \left(W_{c} \cdot\left[h_{t-1}, x_{t}\right]+b_{c}\right)
\end{gathered}
$$

La combinación de ambas funciones permite actualizar el estado de la celda según lo propuesto por la Ecuación (4):

$$
C_{t}=f_{t} \cdot C_{t-1}+i_{t} \cdot C_{t}^{\prime}
$$

Finalmente, la salida de la celda está definida por la puerta de salida de acuerdo con la Ecuación (5) y (6):

$$
\begin{gathered}
O_{t}=\sigma\left(W_{o} \cdot\left[h_{t-1}, x_{t}\right]+b_{o}\right) \\
h_{t}=O_{t} \cdot \tanh \left(C_{t}\right)
\end{gathered}
$$

La red $L S T M$ propuesta utiliza el algoritmo de optimización ADAM (Adaptive Moment Estimation) (Kingma \& Lei Ba, 2017), que es una extensión del algoritmo SGD (Stochastic Gradient Descent) y permite actualizar los pesos de la red de forma iterativa en función de los datos de entrenamiento.

\subsection{Caso de estudio: DAMADICS}

Para validar la propuesta de metodología que se presenta en este trabajo se va a utilizar el problema de prueba DAMADICS que representa un actuador electro-neumático inteligente de muy amplio uso en las industrias y representativo de un sistema mecánico (Bartys et al., 2006). Toda la información relacionada con este proceso de prueba y los datos utilizados puede ser encontrado en http://diag.mchtr.pw.edu.pl/damadics/. Este actuador consta de las siguientes partes:

- Válvula de control

- Servomotor neumático de resorte y diafragma

- Posicionador

La estructura general de este actuador se muestra en la Figura 3.

De manera general, el funcionamiento del actuador es el siguiente: la válvula de control actúa sobre el flujo del fluido que pasa a través de la instalación de la tubería. El servomotor realiza un cambio en la posición del obturador de la válvula de control, actuando sobre el caudal de fluido. El servomotor neumático de resorte y diafragma es un dispositivo accionado por fluido compresible en el que el fluido actúa sobre el diafragma flexible, para proporcionar movimiento lineal del vástago del servomotor. El posicionador ayuda a eliminar las posiciones incorrectas del vástago de la válvula de control producidas por las fuentes externas o internas tales como: fricción, holgura en montajes mecánicos, variaciones de presión de suministro, fuerzas hidrodinámicas, entre otros. En este actuador se diagnosticarán 5 fallos los cuales se muestran en la Tabla 1 .

\begin{tabular}{ll}
\multicolumn{2}{c}{ Tabla 1. Fallos simulados en DAMADICS } \\
\hline Fallo & Descripción \\
\hline 1 & Obstrucción de la válvula \\
7 & Flujo crítico \\
12 & Fallo del transductor electroneumático \\
15 & Fallo del resorte del posicionador \\
19 & Fallo del sensor de caudal \\
\hline
\end{tabular}

Estos fallos fueron escogidos por ser representativos de diferentes partes del actuador y en el caso de los fallos F15 y F19 son fallos que sus patrones se superponen dificultando la clasificación correcta.

Las variables medidas se presentan en la Tabla 2

Tabla 2. Variables medidas en el proceso

\begin{tabular}{lr}
\hline Descripción & Variable \\
\hline Señal externa del control de proceso & $\mathrm{CV}$ \\
Presión en el fluido de ingreso & $\mathrm{P} 1$ \\
Presión en el fluido de salida & $\mathrm{P} 2$ \\
Desplazamiento del vástago & $\mathrm{X}$ \\
Tasa de flujo de líquido & $\mathrm{F}$ \\
Valor del proceso & $\mathrm{PV}$ \\
\end{tabular}

\section{6 Índices para medir el desempeño}

En este trabajo la robustez de la metodología propuesta se considera a partir de lograr un desempeño elevado de clasificación correcta con un bajo porciento de falsas alarmas tal y como se define en Patan (2008). Por lo tanto, para medir el desempeño y la robustez del sistema propuesto a partir de los resultados de la matriz de confusión, se utilizarán los siguientes índices:

Exactitud (Acc): Este indicador evalúa el desempeño general del clasificador. Se define como:

$$
A c c=\frac{V P+V N}{V P+V N+F P+F N}
$$

donde VP (Verdaderos Positivos) representa la cantidad de observaciones pertenecientes a una clase clasificadas correctamente, FP (Falsos Positivos) representa la cantidad de observaciones clasificadas erróneamente como correctas, VN (Verdaderos Negativos) representa la cantidad de observaciones no pertenecientes a una clase clasificadas correctamente como negativas y FN (Falsos 
Negativos) representa la cantidad de observaciones clasificadas como que no pertenecen a una clase y sin embargo si pertenecen a la misma.

Razón de Falsas Alarmas (FAR): Es un indicador importante porque indica la razón de observaciones correspondientes al funcionamiento normal que son clasificadas erróneamente como si fueran algún tipo de fallo y se define como:

$$
F A R=\frac{F P}{V N+F P} 100 \%
$$

\subsection{Diseño de los experimentos}

\subsubsection{Arquitectura de la red LSTM}

Para la aplicación en la propuesta realizada en este trabajo, la red LSTM se forma mediante la conexión en cascada entre varias capas como se muestra en la Figura 4. Primeramente se encuentra una capara de entrada que recibe las 6 variables que se miden en el proceso; una capa $L S T M$ con 500 unidades ocultas con el formato de salida configurado en modo secuencia completa; una capa de tipo dropout para reducir el sobre-entrenamiento y mejorar la capacidad de generalización de la red; una capa totalmente conectada de 6 clases (correspondientes a los 6 estados de funcionamiento del sistema); seguida de una capa con la función exponencial normalizada o función softmax (Bishop, 2006) y una capa de clasificación a la salida.

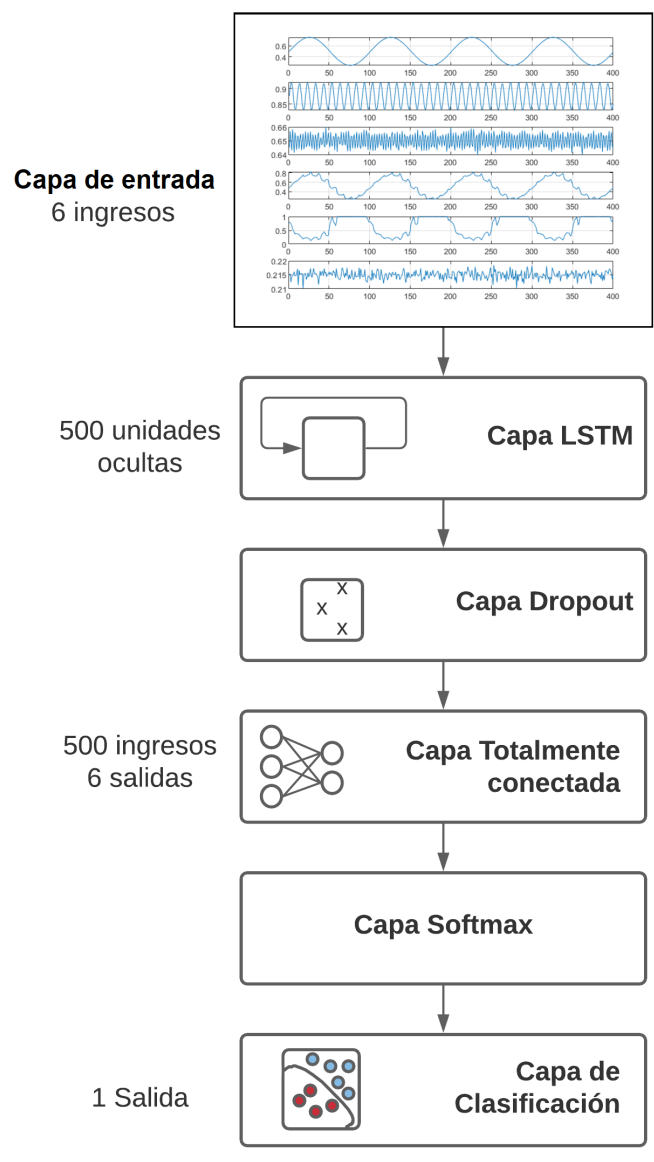

Figura 4. Arquitectura de la red neuronal LSTM empleada

\subsubsection{Entrenamiento de la red}

Una red LSTM se entrena de manera supervisada utilizando un conjunto de secuencias de entrenamiento. Para el entrenamiento se utilizó una combinación entre el algoritmo de optimización $A D A M$ con parámetros $\beta_{1}=0.9$ y $\beta_{2}=0.999$ como fue propuesto en Kingma \& Lei Ba (2017), combinado con el algoritmo BPTT (backpropagation through time) para calcular los gradientes necesarios durante el proceso de optimización, con el fin de cambiar cada peso de la red de LSTM en proporción a la derivada del error en la capa de salida, respecto al peso correspondiente. Como función de activación para actualizar la celda y el estado oculto se usó la función de activación tangente hiperbólica (tanh). El número máximo de épocas utilizadas para el entrenamiento fue de 500 y un umbral de gradiente igual a 2. Para prevenir un sobre entrenamiento de la red se incluyó la capa dropout con una probabilidad de descartar elementos de entrada, especificada como un escalar numérico igual a 0,1 .

La tasa de aprendizaje $(L R)$ es un parámetro importante que influye en el ajuste de pesos y la convergencia de errores. Al elegir una tasa de aprendizaje adecuada, podemos acelerar la convergencia de la red LSTM y mejorar su precisión. En este experimento, la tasa de aprendizaje empleada fue de $L R=0,001$.

Para conformar la matriz de datos para el entrenamiento de la red neuroanl $L S T M$ en la etapa fuera de línea se utilizaron $k=1000$ observaciones de cada una de las $n c=6$ clases $(\mathbf{F N}$ : Funcionamiento Normal, F1: Fallo 1, F7: Fallo 7, F12: Fallo 12, F15: Fallo 15, F19: Fallo 19) almacenadas con un período de muestreo de 1 segundo. En cada observación se leen los valores de las $p=6$ variables descritas anteriormente en la Tabla 2 . Lo anterior indica que la base de datos de entrenamiento está conformada por $n=6000$ observaciones las cuales no tienen ruido asociado ni variables perdidas.

Para el entrenamiento y validación de la red neuronal LSTM propuesta en la etapa fuera de línea se utilizó el proceso de K-validación cruzada (Devijver \& Kittler, 1982). En este caso se seleccionó $K=5$.

Los resultados obtenidos en el proceso de entrenamiento se muestran en la Tabla 3.

Tabla 3. Matriz de confusión para la validación cruzada. Precisión: $97.583 \%$

\begin{tabular}{lrrrrrrrl}
\hline & F1 & F12 & F15 & F19 & F7 & Normal & Acc $(\%)$ & FAR (\%) \\
\hline F1 & $\mathbf{1 9 5}$ & 0 & 0 & 0 & 0 & 5 & & \\
F12 & 0 & $\mathbf{1 9 9}$ & 0 & 0 & 1 & 0 & & \\
F15 & 0 & 4 & $\mathbf{1 9 4}$ & 2 & 0 & 0 & & \\
F19 & 0 & 0 & 2 & $\mathbf{1 9 8}$ & 0 & 0 & & \\
F7 & 1 & 0 & 0 & 0 & $\mathbf{1 9 9}$ & 0 & & \\
Normal & 11 & 0 & 0 & 3 & 0 & $\mathbf{1 8 6}$ & $\mathbf{9 7 , 5 8 3}$ & $\mathbf{0}$ \\
GEN & & & & & & & & \\
\hline
\end{tabular}

\subsubsection{Experimentos a realizar}

Para realizar los experimentos relacionados con la etapa en línea, se utilizó una base de datos con 400 observaciones distintas a las 
usadas en el entrenamiento por cada estado de funcionamiento del sistema por lo que la base de datos para los experimentos estuvo formada por 2400 observaciones. Para garantizar la repetitividad de los resultados cada experimento fue repetido 100 veces y como resultado final se obtuvo el promedio de los resultados obtenidos en esas 100 repeticiones.

Se realizaron los siguientes experimentos para evaluar el desempeño del sistema de diagnóstico con las siguientes características en los datos:

1. Observaciones sin información perdida
a) Sin ruido
b) Con $2 \%$ de ruido
c) Con $5 \%$ de ruido

2. Observaciones con 1 variable perdida por observación de forma aleatoria
a) Sin ruido
b) Con $2 \%$ de ruido
c) Con $5 \%$ de ruido

3. Observaciones con una cantidad aleatoria de variables perdidas entre 0 y 2
a) Sin ruido
b) Con $2 \%$ de ruido
c) Con $5 \%$ de ruido

Para cada experimento, la base de datos con las 2400 observaciones se preparó adecuadamente de acuerdo al tipo de experimento. Para los experimentos con ruido se adicionó a las observaciones un ruido blanco de media cero y con valor del $2 \%$ y del $5 \%$ del valor nominal de cada variable. Para esto se utilizó la relación señal/ruido (SNR) que se define de la siguiente manera:

$$
S N R=10 \cdot \log _{10}\left(\frac{P_{\text {senal }}}{P_{\text {ruido }}}\right)
$$

donde $P_{\text {senal }}$ y $P_{\text {ruido }}$ son la potencia de la señal (variable medida) y del ruido adicionado respectivamente (Russ (2007)).

Para ejemplificar como varía la información de las variables con las variantes previstas en los experimentos y por lo tanto dar una idea de las exigencias a las que se somete la metodología propuesta , la Figura 5 muestra la variable que representa el desplazamiento del vástago en funcionamiento normal, en funcionamiento normal con pérdida de una variable en forma aleatoria e imputación con el valor medio de la misma en el funcionamiento normal y con las mismas condiciones anteriores añadiéndole $2 \%$ y $5 \%$ de ruido. La Figura 6 presenta la misma señal pero ahora con pérdida de información de 0 a 2 variables por observación de forma aleatoria y con $2 \%$ y $5 \%$ de ruido.
Desplazamiento del Vástago (a) Funcionamiento normal

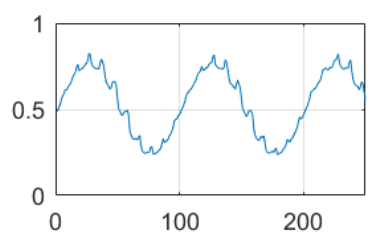

(d) Imputación y $2 \%$ de ruido

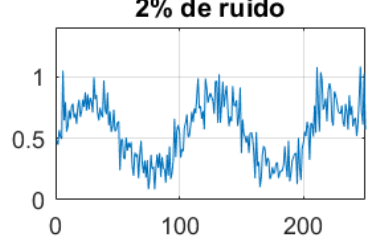

(b) Imputación del promedio 1 dato perdido

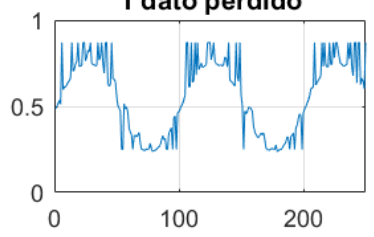

(d) Imputación y $5 \%$ de ruido

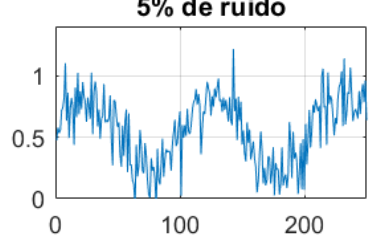

Figura 5. Señal del desplazamiento del vástago utilizada en los experimentos: a) Funcionamiento normal, b) Funcionamiento normal con pérdida e imputación de información, c) Funcionamiento normal con pérdida e imputación de información más $2 \%$ de ruido y d) Funcionamiento normal con pérdida e imputación de información más $5 \%$ de ruido

\section{Desplazamiento del Vástago}

(a) Funcionamiento normal

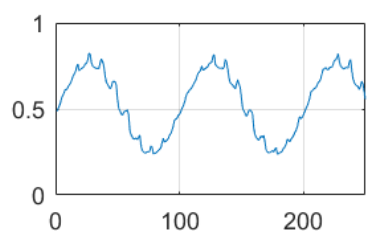

(d) Imputación y

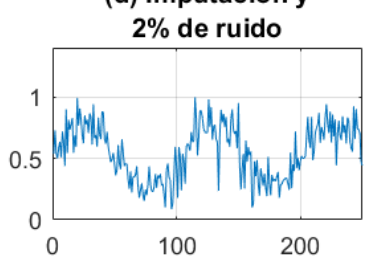

(b) Imputación del promedio

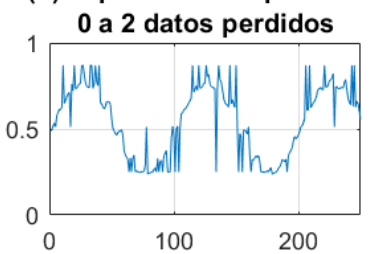

(d) Imputación y $5 \%$ de ruido

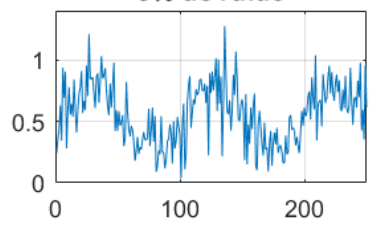

Figura 6. Señal del desplazamiento del vástago utilizada en los experimentos: a) Funcionamiento normal, b) Funcionamiento normal con pérdida de 0 a 2 variables e imputación de información, c) Funcionamiento normal con pérdida de 0 a 2 variables e imputación de información más $2 \%$ de ruido y d) Funcionamiento normal con pérdida de 0 a 2 variables e imputación de información más $5 \%$ de ruido

\section{RESULTADOS Y DISCUSIÓN}

\subsection{Resultados del primer experimento}

La Tabla 4 presenta los resultados del primer experimento.

Como se aprecia el sistema de diagnóstico alcanza un elevado desempeño. Los mayores niveles de confusión se producen entre el Fallo 15 y el 19 lo cual se debe a una cierta superposición que existe entre las dos clases. Además el sistema no genera ninguna falsa alarma $(\mathrm{FAR}=0 \%)$. Estos resultados indican un elevado nivel de robustez ante el ruido de la metodología propuesta. 
Tabla 4. Matriz de Confusión para la clasificación de observaciones sin información perdida (a) Sin ruido (b) Con $2 \%$ de ruido. (c) Con $5 \%$ de ruido

\begin{tabular}{lrrrrrrrl}
\hline (a) & F1 & F12 & F15 & F19 & F7 & Normal & $\operatorname{Acc}(\%)$ & FAR(\%) \\
\hline F1 & $\mathbf{3 9 3}$ & 0 & 0 & 0 & 0 & 7 & & \\
F12 & 0 & $\mathbf{3 9 8}$ & 0 & 0 & 2 & 0 & & \\
F15 & 0 & 9 & $\mathbf{3 9 1}$ & 0 & 0 & 0 & & \\
F19 & 0 & 0 & 22 & $\mathbf{3 7 8}$ & 0 & 0 & & \\
F7 & 4 & 0 & 0 & 0 & $\mathbf{3 9 6}$ & 0 & & \\
Normal & 0 & 0 & 0 & 0 & 0 & $\mathbf{4 0 0}$ & $\mathbf{9 8 , 1 6 7}$ & $\mathbf{0}$ \\
GEN & & & & & & & &
\end{tabular}

\begin{tabular}{lrrrrrrrr}
\hline (b) & & & & & & & & \\
\hline F1 & $\mathbf{3 9 6}$ & 0 & 0 & 0 & 0 & 4 & & \\
F12 & 0 & $\mathbf{3 9 9}$ & 0 & 0 & 1 & 0 & & \\
F15 & 0 & 9 & $\mathbf{3 9 1}$ & 0 & 0 & 0 & & \\
F19 & 0 & 0 & 20 & $\mathbf{3 8 0}$ & 0 & 0 & & \\
F7 & 4 & 0 & 0 & 0 & $\mathbf{3 9 6}$ & 0 & & \\
Normal & 0 & 0 & 0 & 0 & 0 & $\mathbf{4 0 0}$ & $\mathbf{9 8 , 4 1 7}$ & $\mathbf{0}$ \\
GEN & & & & & & & & \\
\hline
\end{tabular}

\begin{tabular}{lrrrrrrll}
\hline (c) & & & & & & & & \\
\hline F1 & $\mathbf{3 9 7}$ & 0 & 0 & 0 & 0 & 3 & & \\
F12 & 0 & $\mathbf{3 9 8}$ & 0 & 0 & 2 & 0 & & \\
F15 & 0 & 9 & $\mathbf{3 9 1}$ & 0 & 0 & 0 & & \\
F19 & 0 & 0 & 18 & $\mathbf{3 8 2}$ & 0 & 0 & & \\
F7 & 3 & 0 & 0 & 0 & $\mathbf{3 9 7}$ & 0 & & \\
Normal & 0 & 0 & 0 & 0 & 0 & $\mathbf{4 0 0}$ & $\mathbf{9 8 , 5 4 2}$ & $\mathbf{0}$ \\
GEN & & & & & & & & \\
\hline
\end{tabular}

\subsection{Resultados del segundo experimento imputando con el valor medio}

En el segundo experimento se eliminó una variable de forma aleatoria a cada observación de la base de datos. El sistema de diagnóstico al recibir cada observación primeramente realiza la clasificación de la observación según el procedimiento presentado en la sección anterior y posteriormente realiza el proceso de imputación con el valor medio de la variable perdida en esa clase.

La Tabla 5 muestra los resultados del segundo experimento.

Los resultados mostrados por la Tabla 5 vuelven a mostrar elevados niveles de desempeño y $0 \%$ de falsas alarmas que indican la satisfactoria robustez de la metodología propuesta ante la imputación por perdida de información y el ruido. Nuevamente el mayor error se produce en la clasificación del fallo 19.

\subsection{Resultados del tercer experimento imputando con el valor me- dio}

Para el experimento 3 se eliminaron de forma aleatoria entre $0 \mathrm{y}$ 2 variables por observación. Cuando el sistema de diagnóstico recibe una observación, primero realiza la clasificación de la observación según el procedimiento presentado en la sección anterior y luego realiza el proceso de imputación con el valor medio de las variables perdidas en esa clase. Posteriormente se realiza la clasificación de la observación. Como en los experimentos anteriores se realizó la imputación y la posterior clasificación para las observaciones sin ruido, con ruido añadido del $2 \%$ y del $5 \%$.

En la Tabla 6 se presentan los resultados obtenidos en el referido experimento.
Tabla 5. Matriz de Confusión para la clasificación de las observaciones con una variable perdida que es imputada con el valor medio (a) Sin ruido, (b) Con $2 \%$ de ruido, (c) Con $5 \%$ de ruido

\begin{tabular}{lrrrrrrrl}
\hline (a) & F1 & F12 & F15 & F19 & F7 & Normal & $\operatorname{Acc}(\%)$ & FAR $(\%)$ \\
\hline F1 & $\mathbf{3 9 3}$ & 0 & 0 & 0 & 0 & 7 & & \\
F12 & 0 & $\mathbf{4 0 0}$ & 0 & 0 & 0 & 0 & & \\
F15 & 0 & 9 & $\mathbf{3 9 1}$ & 0 & 0 & 0 & & \\
F19 & 0 & 0 & 23 & $\mathbf{3 7 7}$ & 0 & 0 & & \\
F7 & 4 & 1 & 0 & 0 & $\mathbf{3 9 5}$ & 0 & & \\
Normal & 0 & 0 & 0 & 0 & 0 & $\mathbf{4 0 0}$ & $\mathbf{9 8 , 1 6 7}$ & $\mathbf{0}$ \\
GEN & & & & & & & &
\end{tabular}

\begin{tabular}{lrrrrrrrl}
\hline (b) & & & & & & & & \\
\hline F1 & $\mathbf{3 9 5}$ & 0 & 0 & 0 & 0 & 5 & & \\
F12 & 0 & $\mathbf{4 0 0}$ & 0 & 0 & 0 & 0 & & \\
F15 & 0 & 9 & $\mathbf{3 9 1}$ & 0 & 0 & 0 & & \\
F19 & 0 & 0 & 18 & $\mathbf{3 8 2}$ & 0 & 0 & & \\
F7 & 3 & 0 & 0 & 0 & $\mathbf{3 9 7}$ & 0 & & \\
Normal & 0 & 0 & 0 & 0 & 0 & $\mathbf{4 0 0}$ & $\mathbf{9 8 , 5 4 2}$ & $\mathbf{0}$ \\
GEN & & & & & & & & \\
\hline
\end{tabular}

\begin{tabular}{lrrrrrrrr}
\hline (c) & & & & & & & & \\
\hline F1 & $\mathbf{3 9 5}$ & 0 & 0 & 0 & 0 & 5 & & \\
F12 & 0 & $\mathbf{4 0 0}$ & 0 & 0 & 0 & 0 & & \\
F15 & 0 & 9 & $\mathbf{3 9 1}$ & 0 & 0 & 0 & & \\
F19 & 0 & 0 & 20 & $\mathbf{3 8 0}$ & 0 & 0 & & \\
F7 & 3 & 0 & 0 & 0 & $\mathbf{3 9 7}$ & 0 & & \\
Normal & 0 & 0 & 0 & 0 & 0 & $\mathbf{4 0 0}$ & $\mathbf{9 8 , 4 5 8}$ & $\mathbf{0}$ \\
GEN & & & & & & & & \\
\hline
\end{tabular}

Tabla 6. Matriz de Confusión para la clasificación de las observaciones con una cantidad aleatoria entre 0 y 2 variables perdidas (a) Sin ruido (b) Con $2 \%$ de ruido (c) Con $5 \%$ de ruido

\begin{tabular}{lrrrrrrrl}
\hline (a) & F1 & F12 & F15 & F19 & F7 & Normal & Acc(\%) & FAR(\%) \\
\hline F1 & $\mathbf{3 9 3}$ & 0 & 0 & 0 & 0 & 7 & & \\
F12 & 0 & $\mathbf{3 9 8}$ & 0 & 0 & 2 & 0 & & \\
F15 & 0 & 10 & $\mathbf{3 9 0}$ & 0 & 0 & 0 & & \\
F19 & 0 & 0 & 11 & $\mathbf{3 8 9}$ & 0 & 0 & & \\
F7 & 4 & 0 & 0 & 0 & $\mathbf{3 9 6}$ & 0 & & \\
Normal & 0 & 0 & 0 & 0 & 0 & $\mathbf{4 0 0}$ & $\mathbf{9 8 , 5 8 3}$ & $\mathbf{0}$ \\
GEN & & & & & & & &
\end{tabular}

\begin{tabular}{lrrrrrrll}
\hline (b) & & & & & & & & \\
\hline F1 & $\mathbf{3 9 2}$ & 0 & 0 & 0 & 0 & 8 & & \\
F12 & 0 & $\mathbf{3 9 9}$ & 0 & 0 & 1 & 0 & & \\
F15 & 0 & 9 & $\mathbf{3 9 1}$ & 0 & 0 & 0 & & \\
F19 & 0 & 0 & 18 & $\mathbf{3 8 2}$ & 0 & 0 & & \\
F7 & 3 & 0 & 0 & 0 & $\mathbf{3 9 7}$ & 0 & & \\
Normal & 0 & 0 & 0 & 0 & 0 & $\mathbf{4 0 0}$ & $\mathbf{9 8 , 3 7 5}$ & $\mathbf{0}$ \\
GEN & & & & & & & $\mathbf{0 8 3 7 5}$ & \\
\hline
\end{tabular}

\begin{tabular}{lrrrrrrrl}
\hline (c) & & & & & & & & \\
\hline F1 & $\mathbf{3 9 2}$ & 0 & 0 & 0 & 0 & 8 & & \\
F12 & 0 & $\mathbf{3 9 8}$ & 0 & 0 & 2 & 0 & & \\
F15 & 0 & 9 & $\mathbf{3 9 1}$ & 0 & 0 & 0 & & \\
F19 & 0 & 0 & 15 & $\mathbf{3 8 5}$ & 0 & 0 & & \\
F7 & 3 & 0 & 0 & 0 & $\mathbf{3 9 7}$ & 0 & & \\
Normal & 0 & 0 & 0 & 0 & 0 & $\mathbf{4 0 0}$ & $\mathbf{9 8 , 4 5 8}$ & $\mathbf{0}$ \\
GEN & & & & & & & & \\
\hline
\end{tabular}

En este experimento, se ratifica la elevada robustez de la metodología propuesta ante la presencia de variables perdidas y ruido y eso indica la excelente capacidad de generalización que logra la red neuronal LSTM.

\subsection{Resultados de los experimentos 2 y 3 imputando con el valor moda}

Experimentos similares a los experimentos 2 y 3 fueron desarrollados pero utilizando como método de imputación el de asignar el valor moda correspondiente a la variable que se va imputar en 
la clase a la que fue asignada la observación analizada. La Tabla 7 muestra los resultados obtenidos.

Tabla 7. Resultados de $\%$ de desempeño y $\%$ de falsas alarmas utilizando como método de imputación el valor moda

\begin{tabular}{lrr}
\hline Experimento & Exactitud (Acc) \% & \% FAR \\
\hline Imputación con la moda & & \\
\hline Datos con 1 variable perdida & $97,15 \%$ & $0 \%$ \\
Datos con 1 variable perdida y 2\% de ruido & $97,78 \%$ & $0 \%$ \\
Datos con 1 variable perdida y 5\% de ruido & $97,59 \%$ & $0 \%$ \\
Datos con 0 2 variables perdidas & $97,60 \%$ & $0 \%$ \\
Datos con 0 a 2 variables perdidas y 2\% de ruido & $98,24 \%$ & $0 \%$ \\
Datos con 0 a 2 variables perdidas y 5\% de ruido & $98,17 \%$ & $0 \%$ \\
\hline
\end{tabular}

Los resultados alcanzados ratifican la robustez del sistema de diagnóstico propuesto.

Para saber si el método utilizado para imputar influye en los resultados que se alcanzaron, se aplicó el test estadístico no paramétrico de Wilcoxon con un nivel de significación $\alpha=95 \%$ para comparar los experimentos similares (Luengo, 2009). Los resultados obtenidos indican que no hay diferencias significativas entre los resultados lo cual indica que cualquiera de los dos métodos se puede utilizar para imputar. Solo debe tenerse en cuenta que en caso de usar el valor medio debe lograrse eliminar previamente en la base de datos de entrenamiento los datos fuera de rango.

\subsection{Comparación con otros resultados presentados en la literatu- ra científica}

Para comparar con otros algoritmos presentes en la literatura científica es necesario desarrollar experimentos con características similares. En toda la literatura científica revisada son pocos los trabajos que abordan el diagnóstico de fallos en presencia de ruido e información perdida y en los encontrados los experimentos fueron desarrollados para otros tipos de sistemas que no son sistemas mecánicos.

En la Tabla 8, se presentan los resultados de técnicas de diagnóstico en sistemas mecánicos publicadas en los últimos 5 años con excelentes desempeños y en ninguno de ellos fue considerado la pérdida de información en las observaciones y solo en algunos casos fue considerado el ruido en las mediciones. Cuando se comparan esos resultados con los obtenidos en este trabajo donde además del ruido hay perdida de información se puede comprobar que los resultados no tienen diferencias significativas lo cual indica la robustez de la metodología que aquí se ha presentado.

Tabla 8. Resultados de la comparación entre algoritmos de la literatura

\begin{tabular}{|c|c|c|c|}
\hline Referencia & Ruido & Datos perdidos & Precisión \\
\hline Li et. al. (2016) & & & $97,68 \%$ \\
\hline Saufi et. al. (2020) & $\mathrm{X}$ & & $99,00 \%$ \\
\hline Medina et. al. (2019) & & & $96,60 \%$ \\
\hline Lee et. al. (2019) & & & $98,66 \%$ \\
\hline Chen et al. (2021) & $\mathrm{X}$ & & $98,46 \%$ \\
\hline
\end{tabular}

\section{CONCLUSIONES}

Los resultados económicos, la seguridad de los operarios y el cuidado del medio ambiente son elementos de gran importancia que hay que tener en cuenta en los requisitos que debe satisfacer la industria moderna. En este trabajo, se propuso una metodología para el diagnóstico de fallos en sistemas mecánicos que consta de una etapa fuera de línea y de otra etapa en línea, que logra elevados niveles de desempeño y robustez en presencia de datos perdidos y ruido en las mediciones. La metodología propuesta realiza la imputación en línea observación a observación en caso de que haya pérdida de información en las observaciones que obtiene el sistema de adquisición de datos, supervisión y control del proceso. Esto es una ventaja con respecto a muchas de las propuestas presentes en la literatura científica donde se necesita acumular un grupo de observaciones antes de realizar la imputación. El proceso de imputación se realiza utilizando el vector de valores medios y el vector de las modas de cada variable en cada clase. Estos métodos de imputación son muy sencillos de implementar y por su baja complejidad computacional permiten su aplicación en procesos con frecuencias de muestreo elevadas, lo cual representa otra ventaja. Para el proceso de clasificación se utilizó una red neuronal de aprendizaje profundo LSTM la cual es entrenada en la etapa fuera de línea logrando una elevada capacidad de generalización, demostrado en los experimentos realizados donde se obtienen altos índices de clasificación satisfactoria y muy bajos porcentajes de falsas alarmas en presencia de variables perdidas y ruido.

Los resultados de la metodología propuesta fueron comparados con otras metodologías presentadas en la literatura científica actual que muestran muy altos porcentajes de desempeño satisfactorio pero que sus resultados no tienen en cuenta la pérdida de información en las observaciones. Los resultados de la comparación demostraron que no existieron diferencias significativas en los altos niveles de clasificación satisfactoria y bajos porcentajes de falsas alarmas logrados. Lo anterior indica el elevado nivel de robustez de la propuesta realizada en este trabajo y por lo tanto las ventajas que ofrece con respecto a otros métodos presentes en la literatura científica.

\section{REFERENCIAS}

Ahmed, Q., Asif Raza, S., Al-Anazi, D.M. (2021). Reliabilitybased fault analysis models with industrial applications: A systematic literature review Quality and $R e$ liability Engineering International, 37(4), 1397-1333. http://dx.doi.org/10.1002/qre.2797

Askarian, M., Escudero, G., Graells, M., Zarghami, R., JalaliFrahani, F., Mostoufi, N. (2016). Fault diagnosis of chemical processes with incomplete observations: A comparative study. Computers \& Chemical Engineering, 84 104-116. http://dx.doi.org/10.1016/j.compchemeng.2015.08.018

Aydin, I., Karakose, M., Akin, E. (2014).An approach for automated fault diagnosis based on a fuzzy decision tree and boundary analysis of a reconstruc- 
ted phase space. ISA Transactions, 53 220-229. Jiang, G., He, H., Yan, J., \& Xie, P. (2019). Multiscahttp://dx.doi.org/10.1016/j.isatra.2013.11.004 le Convolutional Neural Networks for Fault Diagnosis of Wind Turbine Gearbox. IEEE Transactions on Industrial Electronics, 66(4), 3196-3207. https://doi.org/10.1109/TIE.2018.2844805

Bartys, M., Patton, R., Syfert, M., de las Heras, S., Quevedo, J. (2006). Introduction to the DAMADICS actuator FDI benchmark study Control Engineering Practice, 14, 577596. http://dx.doi.org/10.1016/j.conengprac.2005.06.015

Bishop, C.M. (2006). Pattern Recognition and Machine Learning. Springer-Verlag New York. ISBN: 978-0-387-31073-2.

Camps-Echevarría, L., Llanes-Santiago, O., Silva Neto, A.J. (2010). An approach for fault diagnosis based on bio-inspired strategies IEEE Congress on Evolutionary Computation, Barcelona, Spain. http://dx.doi.org/10.1109/CEC.2010.5586357

Cerrada, M., Sánchez, R., Li, C., Pacheco, F., Cabrera, D., de Oliveira, J.V., Vázquez, R.E. (2018). A review on data-driven fault severity assesment in rolling bearings. Mechanical Systems and Signal Processing, 99169-196. http://dx.doi.org/10.1016/j.ymssp.2017.06.012

Cerrada, M., Sánchez, R.V., Pacheco, F., Cabrera, D., Zurita, G., Li, C. (2016). Hierarchical feature selection based on relative dependency for gear fault diagnosis. Applied Intelligence, 44687-703. http://dx.doi.org/10.1007/s10489-015-0725-3

Chen, Y., \& Li, Y. (2018). Computational intelligence assisted design: in industrial revolution 4.0. CRC Press.

Chen, X., Zhang, B. and Gao, D. (2021). Bearing fault diagnosis base on multi-scale CNN and LSTM model. Journal of Intelligent Manufacturing, 32(4), 971-987. https://doi.org/10.1007/s10845-020-01600-2

Devijver, P. A., \& Kittler, J. (1982) Pattern Recognition: A Statistical Approach. Prentice-Hall-London.

Eren, L., Ince, T., \& Kiranyaz, S. (2019). A Generic Intelligent Bearing Fault Diagnosis System Using Compact Adaptive 1D CNN Classifier. Journal of Signal Processing Systems, 91(2), 179-189. https://doi.org/10.1007/s11265-018-1378-3

Eskelson, B.N.I., Temesgen, H., Lemay, V., Barret, T., Crookston, N.L., Hudak, A.T. (2009). The roles of nearest neighbor methods in imputing missing data in forest inventory and monitoring databases. Scandinavian Journal of Forest Research, 24(3), 235-246. https://doi.org/10.1080/02827580902870490

Ger, F.A., Schmidhuber, J., Cumminns, F.(2000). Learning to Forget: continual prediction with LSTM. Neural Computation, $12,2451-2471$.

Hochreiter, S., \& Schmidhuber, J. (1997). Long short-term memory Neural Computation, 9, 125-138.
Kingma, D.P. \& Lei Ba, J. (2017) ADAM: A method for stochastic optimization arXiv:1412.6980v9 [cs.LG] 30 Jan 2017 115 .

Laencina, P.J.G., Gómez, J.L.S., Vida, A.R.F. (2010). Pattern classification with missing data: a review. Neural Computing and Applications, 19(2), 263-282. http://dx.doi.org/10.1007/s00521-009-0295-6

Lee, J. H., Pack, J. H., \& Lee, I. S. (2019). Fault diagnosis of induction motor using convolutional neural network. Applied Sciences (Switzerland), 9(15). https://doi.org/10.3390/app9152950

Li, C., Cabrera, D., Sancho, F., Sánchez, R. V., Cerrada, M., Long, J., \& Valente de Oliveira, J. (2021). Fusing convolutional generative adversarial encoders for $3 \mathrm{D}$ printer fault detection with only normal condition signals. Mechanical Systems and Signal Processing, 147. 107108. https://doi.org/10.1016/j.ymssp.2020.107108

Li, C., Sanchez, R. V., Zurita, G., Cerrada, M., Cabrera, D., \& Vásquez, R. E. (2016). Gearbox fault diagnosis based on deep random forest fusion of acoustic and vibratory signals. $\mathrm{Me}$ chanical Systems and Signal Processing,76-77, 283-293. https://doi.org/10.1016/j.ymssp.2016.02.007

Lin, W. C., \& Tsai, C. F. (2020). Missing value imputation: a review and analysis of the literature (2006-2017). Artificial Intelligence Review, 53(2), 1487-1509. https://doi.org/10.1007/s10462-019-09709-4

Little, R., \& Rubin, D. (2014). Statistical Analysis with Missing Data. John Wiley \& Sons.

Liu, R., Yang, B., Zio, E., \& Chen, X. (2018). Artificial intelligence for fault diagnosis of rotating machinery: A review. Mechanical Systems and Signal Processing, 108 33-47. https://doi.org/10.1016/j.ymssp.2018.02.016

Llanes-Santiago, O., Rivero-Benedicto, B. C., Gálvez-Viera, S. C., Rodríguez-Morant, E. F., Torres-Cabeza, R., \& SilvaNeto, A. J. (2018). A Fault Diagnosis Proposal with Online Imputation to Incomplete Observations in Industrial Plants. Revista Mexicana de Ingeniería Química, 18(1), 83-98. https://doi.org/10.24275/uam/izt/dcbi/revmexingquim/201 $9 \mathrm{v} 18 \mathrm{n} 1 /$ Llanes

Luengo, J., García, S., Herrera, F. (2009). AA study on the use of statistical tests for experimentation with neural networks: Analysis of parametric test conditions and non-parametric tests Expert Systems with Applications, 36 7798-7808. https://doi.org/10.1016/j.eswa.2008.11.041 
Medina, R., Macancela, J. C., Lucero, P., Cabrera, D., Cerrada, M., Sánchez, R. V., \& Vásquez, R. E. (2019). A LSTM Neural Network Approach using Vibration Signals for Classifying Faults in a Gearbox. International Journal of Advanced Manufacturing Technology, 104(5-8), 2195-2214. https://doi.org/10.1007/s00170-019-03858-0

Medina, R., Macancela, J. C., Lucero, P., Cabrera, D., Li, C., Cerrada, M., Sanchez, R. V., \& Vasquez, R. E. (2019). Vibration signal analysis using symbolic dynamics for gearbox fault diagnosis. Proceedings 2019 International Conference on Sensing, Diagnostics, Prognostics, and Control, SDPC 2019, 208-214. https://doi.org/10.1109/SDPC.2019.00045

Patan, K. (2008). Artificial Neural Networks for the modeling and fault diagnosis of technical process. LNCIS, 377, Springer, Berlin.

Prieto-Moreno, A., Llanes-Santiago, O., García Moreno, E. (2015). Principal componentes selection for dimensionality reduction using discriminant information applied to fault diagnosis Journal of Process Control, 3314-24. http://dx.doi.org/10.1016/jprocont.2015.06.003

Raghunathan, T.E., Lepkowski, J.M., Hoewyk, J. V., Solenberger, P. (2001). A Multivariate Technique for Multiply Imputing Missing; Values usisng a Sequence of Regression Models. Survey Methodology 27 Statistics Canada, Catalogue No. 12001, 27(1),85-95.

Rezamand, M., Kordestani, M., Carriveau, R., Ting, D. S. K., \& Saif, M. (2020). A New Hybrid Fault Detection Method for Wind Turbine Blades Using Recursive PCA and WaveletBased PDF. IEEE Sensors Journal 20(4), 2023-2033. https://doi.org/10.1109/JSEN.2019.2948997

Rivera, P.J., Serrano Mercado, E., Llanes-Santiago O., Anido Rifón, L. (2018). Modeling preventive maintenance of manufacturing process with probabilistic Boolean networks with interventions Journal of Intelligent Manufacturing, 29(8), 1941-1952. http://dx.doi.org/10.1007/s10845-016-1226-x

Rodríguez Ramos, A., Bernal de Lázaro, J. M., PrietoMoreno, A., da Silva Neto, A. J., \& Llanes-Santiago, O. (2019). An approach to robust fault diagnosis in mechanical systems using computational intelligence. Journal of Intelligent Manufacturing, 30(4), 1601-1615. https://doi.org/10.1007/s10845-017-1343-1

Rodríguez Ramos, A., Domínguez Acosta, C., Rivera Torres, P. J., Serrano Mercado, E. I., Beauchamp Baez, G., Rifón, L. A., \& Llanes-Santiago, O. (2019). An approach to multiple fault diagnosis using fuzzy logic. Journal of Intelligent Manufacturing, 30(1), 429-439. https://doi.org/10.1007/s10845-016-1256-4

Russ, J.C. (2007) The image processing handbook. CRC Press. ISBN 0-8493-7254-2.
Saufi, S. R., Ahmad, Z. A. Bin, Leong, M. S., \& Lim, M. H. (2020). Gearbox Fault Diagnosis Using a Deep Learning Model with Limited Data Sample. IEEE Transactions on Industrial Informatics, 16(10), 6263-6271. https://doi.org/10.1109/TII.2020.2967822

Sovilj, D., Eirola, E., Miche, Y., Björk, K.M., Nian, R., Akuso, A. (2016). Extreme learning machine for missing data using multiple imputations. Neurocomputings, 174, Part A(22), 220-231. http://dx.doi.org/10.1007/s00521-009-0295-6

Ustundag, A., \& Cevikcan, E. (2017) Industry 4.0: Managing The Digital Transformation. Springer, Cham

Watanabe, K., Matsuura, I., Abe, M., Kubota, M., Himmelblau, D.M. (1989). Incipient fault diagnosis of chemical processes via artificial neural networks. AIChE, 35(11), 18031812.

Xiao, D., Huang, Y., Zhang, X., Shi, H., Liu, C., \& Li, Y. (2019). Fault Diagnosis of Asynchronous Motors Based on LSTM Neural Network. Proceedings - 2018 Prognostics and System Health Management Conference, PHM-Chongqing 20182017, 540-545. https://doi.org/10.1109/PHM-Chongqing.2018.00098

Zhang, D., Zhang, Y., Li, Q., Vance, N., Wang, D. (2018). Robust state prediction with incomplete and noisy measurements in collaborative sensing. Proceedings - 15th IEEE International Conference on Mobile Ad Hoc and Sensor Systems, MASS 2018, 460-468. . https://doi.org/10.1109/MASS.2018.00070

Zabiński, T., Maoczka, T., Kluska, J., Madera, M., \& Sep, J. (2019). Condition monitoring in Industry 4.0 production systems - The idea of computational intelligence methods application. Procedia CIRP, 79, 63-67. https://doi.org/10.1016/j.procir.2019.02.012

\section{BIOGRAFÍAS}

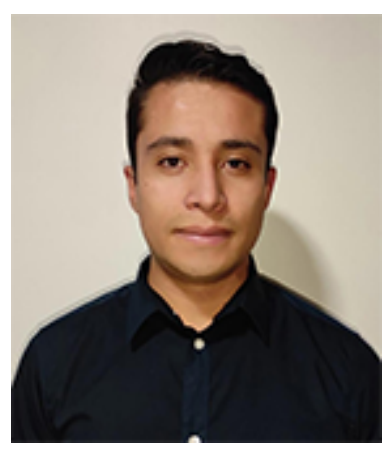

Francisco Javier Ortiz Ortiz. Nació en Cuenca-Ecuador en 1992. Ingeniero Electrónico por la Universidad Politécnica Salesiana en 2016. Candidato a Magíster en Electrónica y Automatización con mención en Informática Industrial por la Universidad Politécnica Salesiana. Desde el 2016 al 2018 formó parte del Grupo de Investigación en Ingeniería Biomédica (GIIB-UPS) en la Universidad Politécnica Salesiana participando con ponencias en congresos internacionales y concursos nacionales de investigación. Actualmente se desempeña como Docente en la UEF Técnico Salesiano de la ciudad de Cuenca y cómo Ingeniero Electrónico en libre ejercicio. 


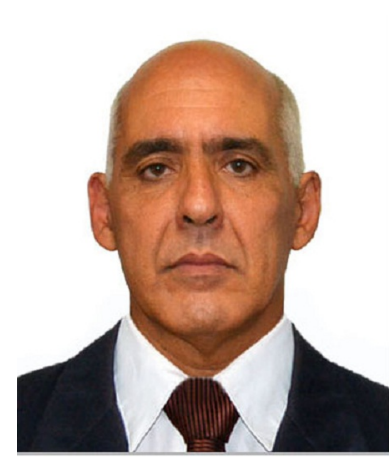

Orestes Llanes-Santiago. Se graduó de Ingeniero Eléctrico en la especialidad de Máquinas Computadoras en la Universidad Tecnológica de La Habana José Antonio Echeverría, CUJAE, en 1981. Desarrolló estudios de postgrado entre 1989 y 1994 en la Universidad de Los Andes, Venezuela, donde obtuvo el grado de Master en Ciencias en Ingeniería de Control en 1990 y el de Doctor en Ciencias Aplicadas mención Control Automático en 1994. Actualmente es Profesor e Investigador Titular del Departamento de Automática y Computación en la CUJAE donde dirige el Laboratorio y el grupo de investigación en Estimación de Parámetros y Diagnóstico de Fallos en Sistemas Industriales. Además, es Académico Titular de la Academia de Ciencias de Cuba, Editor Jefe de la Revista Ingeniería Electrónica, Automática y Comunicaciones (RIELAC) y editor asociado de la Revista Iberoamericana de Automática e Informática Industrial (RIAI). Sus intereses de investigación son el diagnóstico de fallos en sistemas industriales, el control no lineal, y la inteligencia computacional con aplicaciones en el control automático de procesos . 